\title{
Regions of cardiorespiratory synchronization in humans under paced respiration
}

\author{
S. Rzeczinski, N. B. Janson, A. G. Balanov, and P. V. E. McClintock \\ Department of Physics, Lancaster University, Lancaster, LA1 4YB, United Kingdom
}

(Received 21 June 2002; published 19 November 2002)

\begin{abstract}
Cardiorespiratory synchronization under paced respiration is studied systematically as the respiration frequency is changed between 3 and 30 breaths per min. We plot a one-dimensional cut of the classical picture of synchronization regions along the line defining the current breathing amplitude. The existence of $n: m$ synchronization regions of finite width is demonstrated for each of six subjects studied. The statistics of the different types of synchronization and their stability are discussed.
\end{abstract}

DOI: 10.1103/PhysRevE.66.051909

\section{INTRODUCTION}

It has long been known that cardiorhythm is influenced by respiration, a phenomenon called respiratory sinus arrhythmia (RSA) [1-4]. RSA is a modulation of the heart rate resulting from interaction between the processes of respiration and heart rhythm generation. This interaction can sometimes cause the related but different phenomenon of synchronization [5-9]. Cardiorespiratory synchronization (CRS) manifests itself through entrainment between heart and respiration rates [10-13], and has been shown to be useful for medical diagnostics in humans [14] and in rats [15].

RSA has been widely studied, both in freely breathing subjects, and in subjects performing paced respiration where breathing is synchronized with some external signal $[16,17]$. Paced respiration is now a well-established tool for relaxation and for the treatment of chronic pain and insomnia, dental and facial pain, etc. [18-20]. Modulation of heart rate due to paced respiration was systematically studied in Refs. [21,22]: in Ref. [21] three values of respiration frequency, and in Ref. [22], the frequency range between $0.083 \mathrm{~Hz}$ to $0.2 \mathrm{~Hz}$, were examined. CRS has been detected in subjects undergoing paced respiration [23] and the cardiorespiratory interaction has been used as a diagnostic tool [24]. In clinical practice, and occasionally in everyday life, the need can arise to control the heart rate without involving invasive or complex techniques. It is known that a change of respiration parameters can lead, not only to modulation of the heart rate, but also to a shift in its average value. In this context paced respiration would appear to be a promising technique for the control of heart rate. However, there have been no systematic studies of the synchronization of heart rate by paced respiration, and a number of important questions remain open. For example, it is important to know whether CRS is stable under variation of the frequency of paced respiration. Does this phenomenon occur randomly, or are there ranges of paced respiration frequency when synchronization is to be expected with a larger probability, in spite of noise and nonstationarity? What types of $n: m$ synchronization appear most frequently during paced respiration? The present work is an attempt to answer these questions.

From the viewpoint of oscillation theory, the cardiovascular system (CVS) can be treated as a self-sustained oscillator whose basic frequency is the average heart rate, and where respiration can be regarded as an external forcing. According to theory, the average frequency of self-oscillation can
PACS number(s): 87.19.Hh, 87.80.Vt, 87.80.Tq, 05.45.Xt

change whenever external forcing is applied, regardless of whether or not synchronization takes place. However, synchronization preserves a constant ratio between the forcing and response frequencies while either the forcing or the system parameters are varied. Thus, to predict the effect of forcing, one needs to know in detail how the CVS responds to a change in the parameters of forcing, the latter being paced respiration. In mathematical terms, one needs a bifurcation diagram (map of regimes) in a plane of the control parameters, for example, the respiration-amplitude versus respiration-frequency plane, delineating the synchronous and asynchronous regimes for every individual. This is an idealized and ambitious goal which will not be addressed here in view of the unavoidable restrictions imposed on the study of any living system. The more modest aim of the present work is to reveal a one-dimensional cut of the complete map of regimes defined by the frequency of paced respiration. Even a rough picture of this kind, showing the transitions through different synchronous and asynchronous regimes as just one control parameter is increased, can provide some indication of the global structure.

In contrast to experiments with, e.g., electronic oscillators, the parameters of a living system cannot be kept constant but continuously vary. The CVS is influenced by a huge number of factors that change in time. Such changes cannot be eliminated, even when the subject is fully relaxed, but constitute unavoidable manifestations of noise and nonstationarity. In this situation, even the existence of synchronization that is stable under variation of forcing parameters becomes questionable.

The structure of this paper is as follows. In Sec. II basic definitions are given and assumptions about the system under study are formulated, allowing the problem to be considered in terms of oscillation theory. Section III describes experiments. Section IV describes the techniques used for data processing. The results obtained are given in Sec. V. Section VI contains a discussion and Section VII some conclusions.

\section{THEORETICAL BACKGROUND}

Synchronization between two or more interacting oscillatory systems is a fundamental phenomenon [5-9]. For two interacting, self-sustained (dissipative, nonlinear), systems it manifests itself as an adjustment of their basic frequencies $f_{1}, f_{2}$ so as to satisfy the relation $n f_{1}=m f_{2}$, where $n$ and $m$ are integers. Synchronization with the given values of $n$ and 


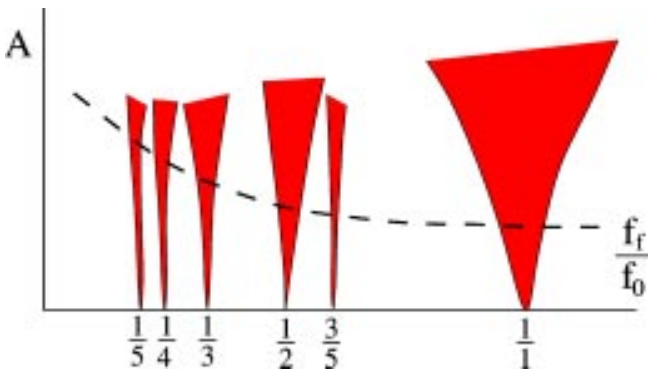

FIG. 1. Sketch of the structure of the synchronization (Arnold) tongues on the parameter plane: frequency-ratio versus forcing amplitude. The dashed line shows the kind of one-dimensional cut through this map of regimes that was sought in the experiments.

$m$ is called " $n: m$ synchronization." Another manifestation of synchronization is adjustment of the phases $\varphi_{1}(t)$ and $\varphi_{2}(t)$ of the interacting systems so as to satisfy

$$
|| n \varphi_{1}(t)-m \varphi_{2}(t)|-C|<\epsilon,
$$

where $t$ is (continuous) time, $C$ is some constant and $\epsilon$ $<2 \pi$. Synchronization can occur either when the two systems mutually influence each other (bidirectional coupling), or when one system forces the other without feedback (unidirectional coupling). According to the theory of synchronization of periodic [25] (and even chaotic) oscillations, synchronization occurs when certain conditions on both the frequency and amplitude of forcing are satisfied. Let the basic frequency of unforced oscillations in the system be $f_{0}$, the amplitude and frequency of forcing be $A$ and $f_{f}$, respectively, and the basic frequency of forced oscillations be $f$. The quantity $\Delta f=\left(f_{f} / f_{0}\right)-(n / m)$ is the frequency detuning between the system and the forcing, and $f_{f} / f_{0}$ is their frequency ratio. Then, the larger $\Delta f$ is, the larger the $A$ that is needed to synchronize the system. For a periodic system forced periodically, a common structure for the $n: m$ synchronization regimes in the "forcing amplitude"- "frequency ratio" parameter plane, for relatively small values of $A$, is shown in Fig. 1 [25]. Synchronization regions have the shape of horns. They are now commonly referred to as Arnol'd tongues. Each tongue begins with a point on the abscissa axis which is equal to the corresponding $n: m$ value. For weak forcing, the larger the value of $m$, the narrower is the synchronization tongue, at least for $m>4$ [26]. For weak forcing the transition from an asynchronous to a synchronous regime is associated with the transition from an ergodic to a resonant torus in the phase space. If $m<4$, the synchronization is called a "strong resonance," and if $m \geqslant 4$ the resonance is called "weak." A very important feature of the synchronization phenomenon is its robustness: that is, if one is inside the synchronization tongue, a small variation of $\Delta f$ and (or) or $A$ should not lead to disappearance of the effect. A qualitatively similar picture of synchronization regions was also observed for chaotic [27] and even for stochastic [28] oscillations.

If the interacting systems are periodic and subject to noise [29], or chaotic (maybe, also with noise) [30], Eq. (1) can only remain satisfied during finite time intervals. In this case, there is no strict definition of either synchronization or synchronization regions. Whether or not to treat an observed regime as synchronous depends solely on the judgement of the observer. Usually, a regime is considered to be synchronous when the duration of the plateau defined by Eq. (1) exceeds some predefined number of basic oscillation periods. Synchronization persisting only during a finite time interval is known as effective synchronization [31]. Following this notation, we will hereinafter refer to the average relative duration of synchronization epochs as the effectiveness of the synchronization, for brevity.

It is known that an isolated heart can continue to contract rhythmically for a long time at a very stable frequency, that is, it behaves as a self-sustained oscillator. It is also known that respiration affects heart rate much more strongly than the heart activity affects respiration [32]. Thus, as a good approximation, one can assume that respiration forces heart rate in a unidirectional manner. This forcing can be easily brought very close to being periodic through paced respiration. With these assumptions, we can treat the CVS of a human undergoing paced respiration as a generator under periodic forcing, affected by noise and nonstationarity. Synchronization theory should therefore be applicable.

We change the frequency of paced respiration gradually between the feasible extreme frequencies of $0.05-0.5 \mathrm{~Hz}$. It is known ( [32], and references therein) that normally, the smaller the breathing frequency, the larger its amplitude, since the average volume of air required to go through the system per unit time is roughly the same. We aim to establish whether $n: m$ synchronization between respiration and heart rate took place for the given respiration frequency by comparing the forcing and response signals. In this way, we aim to plot a one-dimensional cut of the map of regimes along the curve formed by the current values of forcing amplitude, sketched in Fig. 1 as a dashed line.

\section{DESCRIPTION OF MEASUREMENTS}

Six volunteers participated in the measurements. They were young men aged 22-29 years, non-smokers, without any history of cardiopulmonary disease. The sessions were performed 3-4 $\mathrm{h}$ after the last meal, and no caffeinated beverage was taken on the day of the experiment.

Each subject was asked to lie on a bed in a comfortable position, with his head on a pillow, slightly tilted. An interval of about 20 min followed in order to achieve full relaxation. Values of heart rate were measured and compared several times during this period, to establish that relaxation had taken place. A signal with which to synchronize respiration was generated by computer and presented to the subject as a series of light and sound pulses of duration $0.1 \mathrm{sec}$ as described in Ref. [33]: he was able to see a red square on the computer screen and hear the sound. He was asked to inhale when the sound and light signal appeared, and to exhale at his convenience before being prompted to inhale again by the next signal. We did not impose any restrictions on the amplitude of breathing, and suggested to subjects that they should breathe to whatever depth felt most comfortable for the given frequency.

After about $20 \mathrm{sec}$, the subject was breathing as prescribed by the generated signal and had become accustomed 
to the regime being imposed. Simultaneous measurements of the ECG and the respiration signals were then initiated, and continued for $180 \mathrm{sec}$. The respiration signal was measured using a respiratory effort transducer by Biopac Systems Inc., and the ECG signal by positioning three electrodes on the right and left shoulder and on the last rib on the subject's left.

For each new measurement, the respiration frequency $f_{\text {resp }}$ was increased by 1 breath per min (i.e., by $1 \mathrm{~min}^{-1}$ ). The minimal and maximal respiration frequencies were selected experimentally to be 3 and $30 \mathrm{~min}^{-1}$, respectively, so that they should not be too difficult for the subject. Thus, 28 dataset pairs were measured for each subject. In between measurements the subject was allowed to relax for about 5 min until his heart rate, averaged over $1 \mathrm{~min}$, had stopped changing. The average heart rate at rest was recorded to provide the current value of $f_{0}$ before each new measurement.

The measurements for each subject were completed in a single continuous session (except for subject 2) lasting for about $4 \mathrm{~h}$ in total. This procedure was adopted because, to plot a map of regimes, we were interested in ensuring that the experimental conditions (weather, room temperature, time of the day, etc.) and the physical and mental state of the subject, remained as constant as possible. The relatively large number of datapoints needed to create the map of regimes, and the necessity of completing the measurements for a given subject in one session, imposed restrictions on the time per measurement. On the one hand, the time of one measurement should not be too short. In practice, however, our preliminary experiments have shown that a session cannot reasonably exceed 3-4 h, because a longer time is exhausting for the volunteer. Compromise was obviously necessary, and 3 min was selected as being the optimal time per measurement.

For any given subject, the respiratory effort transducer and ECG electrodes were positioned once at the beginning of the measurements, and not adjusted subsequently during the rest of the session. We can therefore make meaningful comparisons between the relative amplitudes of respiration throughout the whole session for that particular subject.

For two of the six subjects we repeated the measurements several days after the first session. This was done in order to learn whether the effect of cardiorespiratory synchronization can easily be reproduced when the experimental conditions and the state of the subject (mood, food taken, previous activities, etc.) would all have been slightly different.

The first session with subject 2 was an exception, however: it was split into two parts. Measurements for respiration frequencies $f_{\text {resp }}$ of $3-15 \mathrm{~min}^{-1}$ inclusive were completed on the first day, and those for $f_{\text {resp }}$ of $16-30 \mathrm{~min}^{-1}$ inclusive were done on the next day.

\section{DATA PROCESSING TECHNIQUES}

The ultimate goal of our study was to plot a curve split into segments of nonsynchronized and $n: m$ synchronized activity on the plane of the two control parameters $f_{\text {resp }} / f_{0}$ and $A_{\text {resp }}$ (see Fig. 1 for a sketch of the curve), where $A_{\text {resp }}$ and $f_{\text {resp }}$ are the amplitude and frequency of paced respiration, respectively. Following the representation in Fig. 1, we chose to normalize the respiration frequency $f_{\text {resp }}$ to the average heart rate before measurement $f_{0}$, for two reasons. First, the average heart rate in relaxation differs between subjects, implying that the same respiration frequency can lead to different synchronization orders in them. Normalization allows us to compare the synchronization regions of different subjects. Second, the average heart rate changes slightly in the course of experiments, in spite of all precautions, so that $f_{0}$ will not be quite constant in a given subject before different measurements within the same session.

The ratio of the two numbers $f_{\text {resp }}$ and $f_{0}$ is readily computed to find the coordinate on the horizontal axis. The coordinate $A_{\text {resp }}$ on the vertical axis is the amplitude of the respiration signal. Strictly speaking, its amplitude was not constant from one breath to the next. By amplitude we therefore mean an average amplitude. We define $A_{\text {resp }}$ as the square root of the signal power, assessed by integration over its Fourier power spectrum. A one-dimensional cut of the map of regimes is thus straightforward to plot.

The next step is to split this curve into segments corresponding to synchronized and nonsynchronized regimes. The absence or presence of $n: m$ phase synchronization is detected by introducing phases for both respiration and ECG and computing the phase difference

$$
\Delta \varphi(t)=\frac{n}{m} \varphi_{\mathrm{ecg}}(t)-\varphi_{\mathrm{resp}}(t)
$$

In order to establish the values of $n$ and $m$, we first find a rough approximation of the synchronization order $n: m$ by computing the Fourier spectra of the respiration and ECG signals and finding the ratio of their basic frequencies $\xi$ $=f_{\text {resp }} / f_{\text {ecg }}$. After that we try several values of $n$ which, being multiplied by $\xi$ will be close to some integer value $m$. In doing so, we try to find $n$ and $m$ values that are not larger than 15 because, according to the theory, the larger the denominator of the synchronization order, the narrower is the synchronization tongue. In the presence of noise (which is inevitable in real systems) these tongues will be smeared. Furthermore, the relatively rough sampling provided by our 28-point curve made it quite improbable that points would fall within the narrow tongues.

The presence of $n: m$ synchronization was arbitrarily defined by the condition that

$$
|\Delta \varphi(t)-C|<\frac{\pi}{m},
$$

during 5 periods of respiration, or longer. In practice, however, for all respiration frequencies larger than $0.25 \mathrm{~Hz}$ $\left(15 \mathrm{~min}^{-1}\right)$ we seek for plateaus lasting $20 \mathrm{sec}$ or longer. There may be several such plateaus during the same measurement. If at least one plateau of sufficient duration is detected, we attribute that measurement to the corresponding $n: m$ synchronization region.

Sometimes (but rarely) during the same measurement switchings between synchronization regimes of different orders $n: m$ occur. We then attribute the same measurement to two different synchronization tongues. Where several successive values of respiration frequency lead to the same syn- 


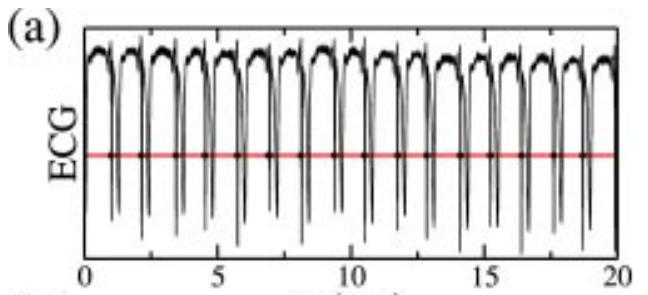

(b)

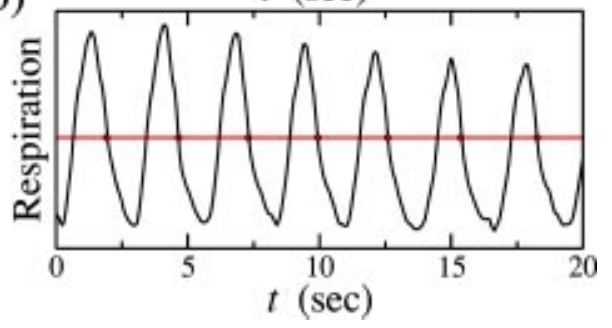

FIG. 2. Illustration of the first method of phase definition, using Eq. (4). (a) ECG of subject No. 4 breathing with frequency $21 \mathrm{~min}^{-1}(0.35 \mathrm{~Hz})$. (b) The corresponding respiration signal. Both signals are given in arbitrary units. Horizontal lines indicate the chosen threshold levels. In each case, the filled circles show intersections of the signal with the level from above, at the moments of time $t_{i}^{\text {ecg }}$ in (a) for the $R$ peak in the ECG, and $t_{i}^{\text {resp }}$ in (b) for the respiration signal, respectively.

chronization order $n: m$, they form the required segment of the curve belonging to the same tongue.

There are several different ways for introducing phases from one-dimensional signals [34]. One way is to store all time moments $t_{i}$ when the signal $x(t)$ crosses some threshold level $\theta$ in one direction (e.g., from above to below) and then attribute to each such crossing a phase increase of $2 \pi$. An illustration of this technique is given in Fig. 2, where an ECG of one of our healthy volunteers [Fig. 2(a)] is given together with his simultaneously measured respiration signal [Fig. 2(b)]. For the ECG, following the usual convention, we count only intersections of the so-called $R$ peak [the highest and narrowest peak, negative going as plotted in Fig. 2(b)] with the threshold. If threshold levels are crossed at the time moments $t_{i}^{\text {ecg }}$ and $t_{i}^{\text {resp }}$, the phases for ECG and respiration are defined as follows:

$$
\begin{gathered}
\left.\left.\varphi_{\mathrm{ecg}}(t)=2 \pi \frac{t-t_{i}^{\mathrm{ecg}}}{t_{i+1}^{\mathrm{ecg}}-t_{i}^{\mathrm{ecg}}}+2 \pi i, \quad t \in\right] t_{i}^{\mathrm{ecg}} ; t_{i+1}^{\mathrm{ecg}}\right] \\
\left.\left.\varphi_{\text {resp }}(t)=2 \pi \frac{t-t_{i}^{\mathrm{resp}}}{t_{i+1}^{\mathrm{resp}}-t_{i}^{\mathrm{resp}}}+2 \pi i, \quad t \in\right] t_{i}^{\mathrm{resp}} ; t_{i+1}^{\mathrm{resp}}\right],
\end{gathered}
$$

where $i$ is the number of the crossing.

Another method is to reconstruct the phase portrait from a signal, project it onto the phase plane and introduce phase as a phase angle on this plane. For the reconstruction of the phase plane one can use various methods, including the Hilbert transform [7] and delay embedding [35]. Delay embedding is easier to implement and requires much less computation time and resources than the Hilbert transform. It is known that for an (almost) periodic signal, the optimal time delay $\tau$ is equal to a quarter of a period. In Fig. 3, delay

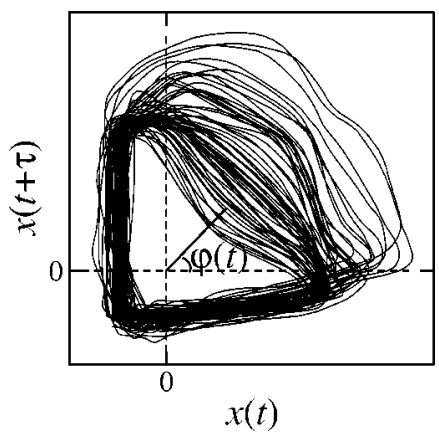

FIG. 3. Projection onto the phase plane of the phase portrait, restored by delay embedding from the respiration signal shown in Fig. 2(b). The signal is given in arbitrary units. The time delay $\tau$ is equal to a quarter of the respiration period and $\varphi(t)$ is the phase angle.

embedding of the respiration signal shown in Fig. 2(b) is illustrated for the optimal time delay. The phase of respiration is then

$$
\varphi_{\text {resp }}(t)=\arctan _{2} \frac{x_{\text {resp }}(t+\tau)}{x_{\text {resp }}(t)}+2 \pi i,
$$

where, emulating Fortran, we denote by $\arctan _{2}$ the $\arctan$ function unwrapped from the interval $[-\pi ; \pi]$ (rather than the conventional $[-\pi / 2 ; \pi / 2]$ interval); $i$ is the number of complete revolutions which have taken place by the time $t$. In our study we introduced phase for the ECG using Eq. (4), and tried both Eqs. (4) and (5) for respiration signal. As expected, the results obtained were independent of the method by which phase was introduced for the respiration signal.

\section{RESULTS}

In Fig. 4 values of $R-R$ intervals are given for all sessions of measurements for all six subjects. The horizontal axis is split by vertical lines into segments of duration $180 \mathrm{sec}$, being the duration of one measurement. $R-R$ intervals within separate neighboring measurement are also marked by different shades to help to distinguish between them. The integers beneath segments mark the frequency of paced respiration in breaths per minute. In the first plot for subject 2 one can see a discontinuity between the measurements for respiration frequencies 15 and $16 \mathrm{~min}^{-1}$. This was due to the break of 1 day between these measurements. All other sets of measurements were done in single sessions, and there are no abrupt jumps in the series of $R-R$ intervals between successive measurements.

By average heart rate we will mean the reciprocal of the average $R-R$ interval during the time of one measurement. Figure 5 plots (a) the average values and (b) the variance of the $R-R$ intervals shown in Fig. 4, as functions of the respiration frequency. Designations for the different subjects are given in the figure caption. It is interesting to note that, with the exception of subject 2, average heart rate either did not change much with increasing respiration frequency (subject 4 ), or it slightly decreased. In subject 2, the average heart rate increased markedly during the first half of the session, 

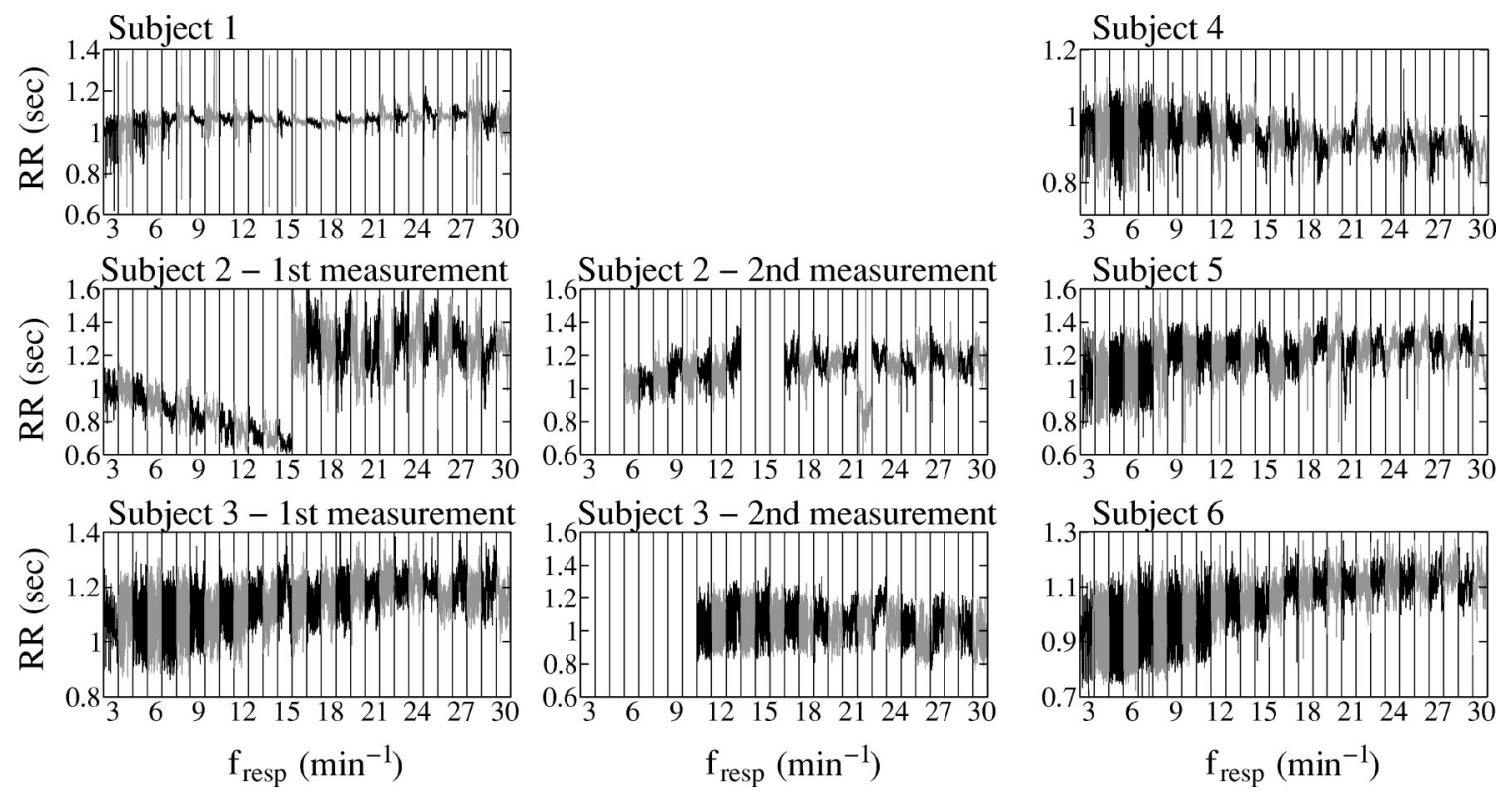

FIG. 4. $R-R$ intervals for all measurement sessions with different frequencies of paced respiration, for all six subjects. The horizontal axes are split by vertical lines into segments of duration $180 \mathrm{sec}$ (the time of one measurement). The integers below segments mark the corresponding frequency of paced respiration in breaths per minute.

the average value of the $R-R$ interval decreasing almost linearly. However, in the second half of the session the behavior of the average heart rate is qualitatively the same as for the other subjects. An increase in the frequency of paced respiration led to a decrease in heart rate variability in all subjects.

In Fig. 6(a) the amplitude of the respiration signal $A_{\text {resp }}$ is plotted as a function of respiration frequency $f_{\text {resp }}$ for all six subjects, who are indicated by different symbols. The respiration amplitude is expressed in arbitrary units and its absolute magnitude is not comparable between different subjects. As predicted, the larger the frequency of breathing, the smaller its amplitude is, on average. In Fig. 6(b) $A_{\text {resp }}$ is shown as a function of the frequency ratio $f_{\text {resp }} / f_{0}$. We use the same designations for individual subjects in Figs. 6(a) and 6(b) as those in Fig. 5.

Some typical plots of phase difference $\Delta \varphi(t)$ are shown in Figs. 7(a), 7(b), and 7(c). Horizontal lines mark the limits of the plateaus defined by Eq. (3). In Fig. 7(a) $\Delta \varphi(t)$ is shown for subject number 4 breathing with frequency $26 \min ^{-1}(0.433 \ldots \mathrm{Hz})$ for $n: m=2: 5$. One can observe a $75 \mathrm{sec}$ plateau, demonstrating the presence of $2: 5$ phase synchronization between respiration and heart rate. Figure 7(b)
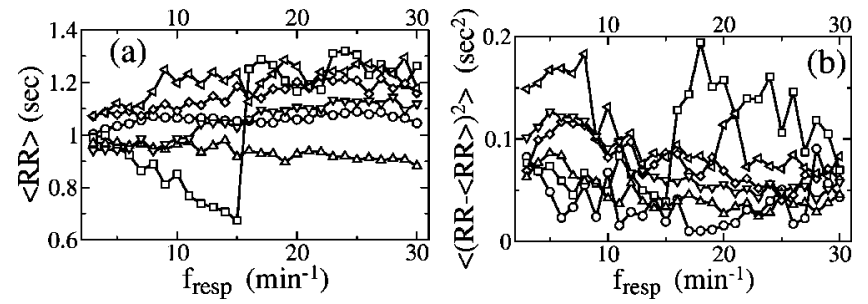

FIG. 5. (a) Average value and (b) variance of the $R-R$ intervals shown in Fig. 4 for the first or only measurements for: subject No. 1 (circles); 2 (squares); 3 (diamonds); 4 (up-pointing triangles); 5 (left-pointing triangles); and 6 (down-pointing triangles). shows $\Delta \varphi(t)$ for subject number 5 breathing with frequency $17 \min ^{-1}(0.2833 \ldots \mathrm{Hz})$ for $n: m=1: 3$. One can see two plateaus lasting $40 \mathrm{sec}$ and $70 \mathrm{sec}$, between which switching occurs. Figure 7(c) shows three plateaus of $\Delta \varphi(t)$ for subject number 4 breathing with the frequency $28 \mathrm{~min}^{-1}$ $(0.4666 \ldots \mathrm{Hz})$. The first and the third segments correspond to $n: m=2: 5$ (black curve), while the second segment corresponds to $n: m=3: 7$ (gray curve). This picture shows that switching from $2: 5$ to $3: 7$ synchronization and back took place during the period of observation.

The right-hand column of Fig. 7 shows the corresponding time dependences of the $R-R$ interval, and of the respiration signal at the moment when the $R$ peak occurs. Thus, stroboscopic sections of the respiration signals are taken at the time moment when the phase of ECG changes by $2 \pi$. In Figs. $7(\mathrm{~d}), 7(\mathrm{e})$, and $7(\mathrm{f})$, the $R-R$ intervals are rescaled so that their dynamics can be compared with that of the respiration signal.

Next, synchronization regions were plotted for each of the six subjects (Fig. 8). In the first column, data for subjects 1 , 2 , and 3 are presented for the first (or only) measurement
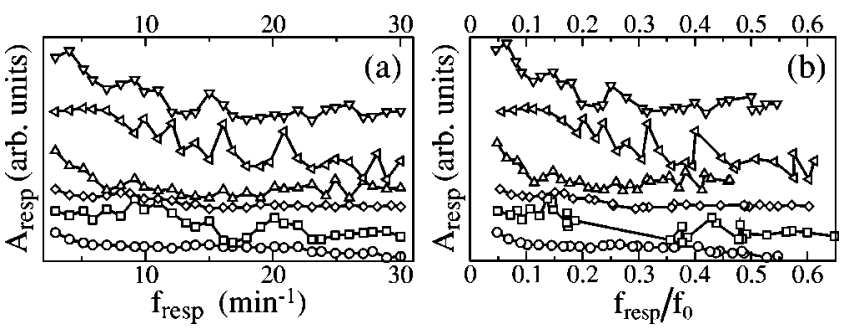

FIG. 6. Amplitude of respiration versus (a) respiration frequency in breaths per minute $\left(\mathrm{min}^{-1}\right)$; (b) ratio of respiration frequency $f_{\text {resp }}$ to the average heart rate before measurement $f_{0}$, for the first or only measurements with all six subjects. Designations of the symbols are the same as in Fig. 5. 

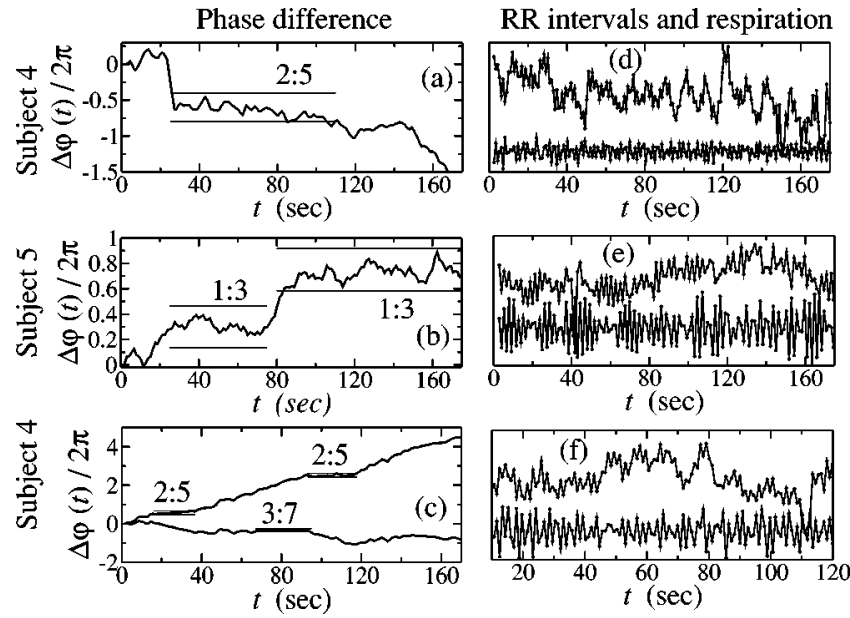

FIG. 7. Left-hand column: typical plots of phase difference $\Delta \varphi(t)$ for different frequencies of paced respiration. (a) $2: 5$ phase synchronization during $75 \mathrm{sec}$ for $f_{\text {resp }}=26 \mathrm{~min}^{-1}$; (b) $1: 3$ phase synchronization during $150 \mathrm{sec}$ for $f_{\text {resp }}=17 \mathrm{~min}^{-1}$; (c) transitions between 2:5 and 3:7 synchronization for the same frequency of paced respiration for $f_{\text {resp }}=28 \mathrm{~min}^{-1}$. Horizontal lines mark the limits defined by condition (3). Right-hand column: plots of $R R$ intervals (above) and the values of respiration signal (below) at the time moments when the $R$ peak occurs, that is, stroboscopic sections of respiration. In each row the same measurement is illustrated.

session. The second column shows plots for the second sessions with subjects 2 and 3 . The third column contains data for subjects 4, 5, and 6 .

In each plot of Fig. 8, the horizontal axis gives the value of the paced respiration frequency $f_{\text {resp }}$ divided by average heart rate immediately before measurement $f_{0}$. The vertical axis is the average amplitude of respiration $A_{\text {resp }}$. The dashed line connects the positions of the measurement points marked by circles [which for complete measurements are the same as those shown in Fig. 6(b)]. Empty circles indicate the absence of any synchronization, while filled circles mark synchronous regimes.

From the theory we know that a synchronization region should grow from a point $(n: m)$ at zero forcing amplitude, and as the latter increases, their widths should increase, too. We have no precise information about how the boundaries of the synchronization regions evolve. We only know that, for very small forcing amplitude, they are straight lines rising from the horizontal axis. To estimate roughly the limits of each synchronization region, we connect the theoretical starting point $(n: m)$ on the horizontal axis with the bounding points of the same region on the experimental curve. The experimental synchronization tongues, estimated in this way, are shown as the shaded regions in Fig. 8.

To estimate the effectiveness (see Sec. II) of the synchronization observed under different conditions, we count the total duration $t_{p l}$ of all plateaus of phase difference $\Delta \varphi(t)$ for the particular value of respiration frequency $f_{\text {resp }}$ and synchronization order $n: m$. We denote the observation time (here $180 \mathrm{sec}$ ) as $t_{o b s}$. The ratio $t_{p l} / t_{o b s} \times 100 \%$ gives us the fraction of time during which the heart rate is synchronized by respiration at the given $n: m$. Figure 9 shows the ratio $t_{p l} / t_{o b s} \times 100 \%$ versus frequency ratio $f_{\text {resp }} / f_{0}$ for all subjects. Figure 10(a) summarizes the data of Fig. 9. The vertical axis indicates the relative durations of plateaus for the most frequently encountered orders of synchronization $n: m$, averaged over all subjects and all measurements in which this synchronization occurs. The numbers over the datapoints give the number of subjects who demonstrated this particular synchronization regime.

Finally, we estimate the robustness of synchronization. We compute the tongue width as the difference of frequency ratios $\Delta\left(f_{\text {resp }} / f_{0}\right)$ at its boundaries, for each of the commoner synchronization orders. If the tongue consists of only one point, we define its width as the step in respiration frequency, $1 \mathrm{~min}^{-1}$, divided by the average heart rate prior to paced respiration $f_{0}$. Note, that this is not the tongue width at the same level of respiration amplitude. This estimate reflects the width of synchronization region along the cut of the parameter plane at the current value of frequency, which, in turn, defines the optimal amplitude. Figure 10(b) gives the values of tongue width for the commoner synchronization orders, averaged over all subjects.

\section{DISCUSSION}

Our observation that heart rate variability, as expressed through the variance of $R-R$ intervals, decreases as the respiration frequency increases (Figs. 4,5) is in a good agreement with earlier observations $[1,17]$. The fact that the larger the frequency of paced respiration, the smaller on average its amplitude becomes (Fig. 6), accords with common sense (because of the need to maintain a particular oxygenation level) and is consistent with earlier experiments [32].

The experimental maps of regimes in Fig. 8 are to be compared with the theoretical one in Fig. 1. The qualitative structure of the theoretical Arnold tongues is reproduced in our experiments, notwithstanding the inevitable real-life circumstances affecting the possibility of synchronization. This allows us to infer that the system governing the heart rate of a healthy human can be treated as a generator in a physical sense, and that paced respiration acts as an external periodic forcing of this system. However, the widths of the synchronization tongues do not seem to be as predicted by the theory of a periodic oscillator forced periodically [Fig. 10(b)]. In particular, the synchronization regions found do not exhibit an obvious decrease of the tongue width with the increase of denominator $m$ of synchronization order $n: m$. It is not obvious what rule, if any, governs the width of the tongue. Such a rule may perhaps be hidden because the actual strength with which heart rate generator is forced is not simply proportional to the amplitude of breathing, as measured by our technique, but may be due to some specific features of the nonlinearity of the system. Consistent with the classical theory, however, we failed to find any synchronization regions with $m>10$, in full agreement with predictions about the improbability of falling within them under the given experimental conditions.

Comparison between experiments conducted with the same subjects on different days have shown that, even where the detuning and amplitude are quite close, the structure of 
Subject 1

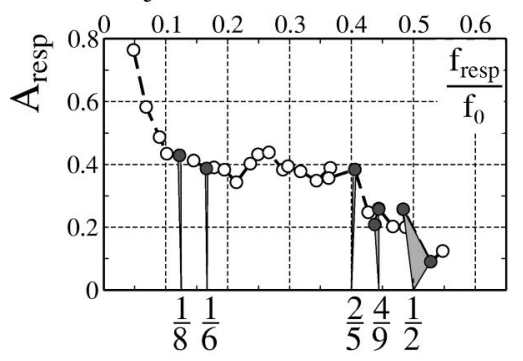

Subject 2 - 1 st measurement

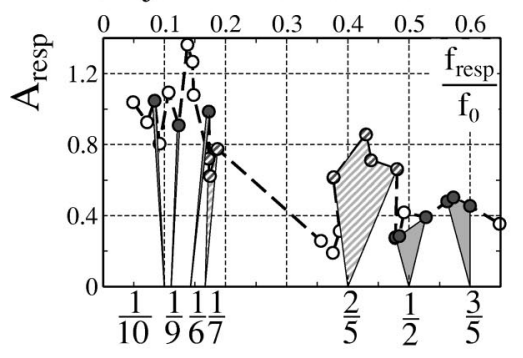

Subject 3 - 1st measurement

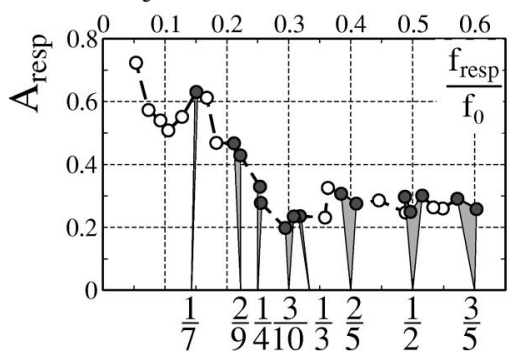

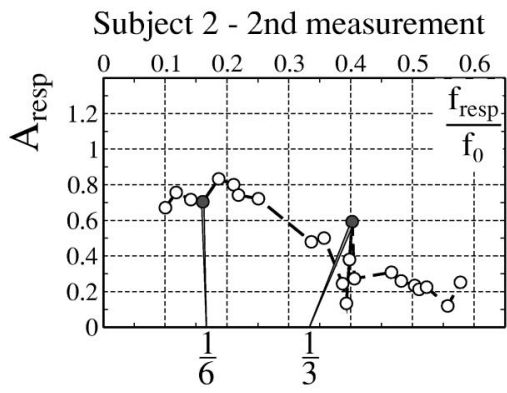

Subject 3 - 2nd measurement

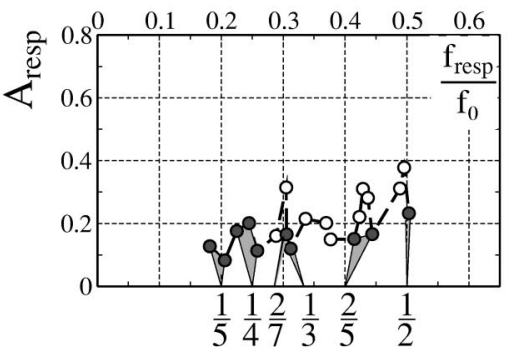

Subject 4

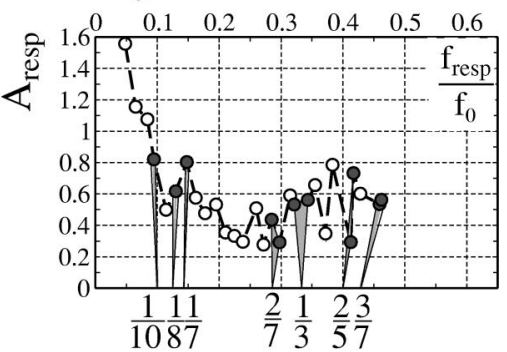

Subject 5

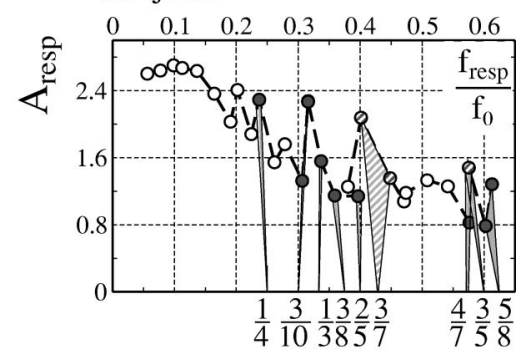

Subject 6

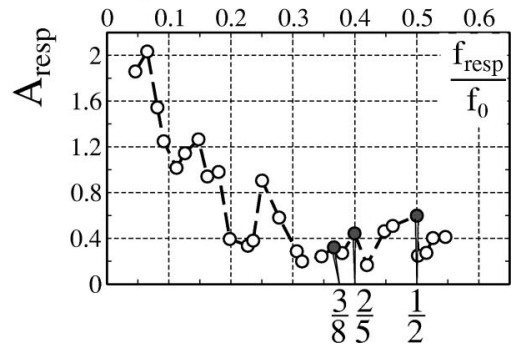

FIG. 8. Regions of cardiorespiratory synchronization for all subjects and all sessions. The first column gives results from the first session with subjects 1,2, and 3; the second column gives results from the second session with subjects 2 and 3; and the third column gives the results for subjects 4, 5, and 6. Dashed lines mark the amplitude of respiration versus the frequency ratio and, for first (or only) sessions, are precisely the same as in Fig. 6(b). Shaded tongues show the inferred synchronization regions, their tips pointing to the corresponding $n: m$ rotation number. These results are to be compared with the predictions of the classical theory as sketched in Fig. 1. Details are given in the text.

the synchronization regions is not precisely reproduced: Subject 3 reproduced four out of eight synchronization regions during his second session; whereas subject 2 reproduced only one of seven synchronization regions. The second sets of measurements also demonstrated synchronization orders that were absent during the first sessions.

Nevertheless, regimes of synchronization with certain $n: m$ are realized in particular subjects more frequently than in others. Namely, 2:5 synchronization was induced in all 6 subjects, $1: 2$ and $1: 3$ in $4,1: 7$ and $3: 5$ in 3. Other types of synchronization appear occasionally. Cardiorespiratory synchronization can be induced with greatest probability in the range of paced respiration frequency $0.15-0.5 \mathrm{~Hz}$.

As to the effectiveness of synchronization of different orders, one can note from Fig. 10(a) that the most effective synchronization was 1:3 in those subjects where it was induced. This means that the average duration of the corresponding plateaus was the longest of all synchronization orders. It is known that the typical range of breathing frequencies in the relaxed state lies between $1 / 6$ and $1 / 3$ of the average heart rate [16]. Note, that the breathing frequency inducing 1:3 synchronization is on the borderline of those which are most common in the relaxed state. Although being the most effective, 1:3 synchronization was encountered in only $2 / 3$ of all subjects. The next most effective regime was 1:2 synchronization, which requires an even less natural breathing frequency. The next regime was 2:5 which was found in all subjects studied. Although being the most prevalent, the 2:5 synchronization appeared to be less effective than the 1:3 and 1:2 ones. Note that the denominator of this synchronization order is larger than 4 which means that this resonance is weak. The corresponding tongue is expected to be narrower than those for $1: 2$ and $1: 3$, which is indeed observed in our experiments. The remaining two common orders of synchronization were $1: 7$ and $3: 5$, both classified as weak resonances. Synchronization of orders 1:6, $1: 5$ and $1: 4$ appeared much more rarely, namely, $1: 4$ and 1:6 in $1 / 3$ and $1: 5$ in $1 / 6$ of all subjects.

\section{CONCLUSIONS}

We note that to draw global conclusions, a larger study will be required, and that it will be interesting to study how the synchronization phenomena depend on, for example, the 

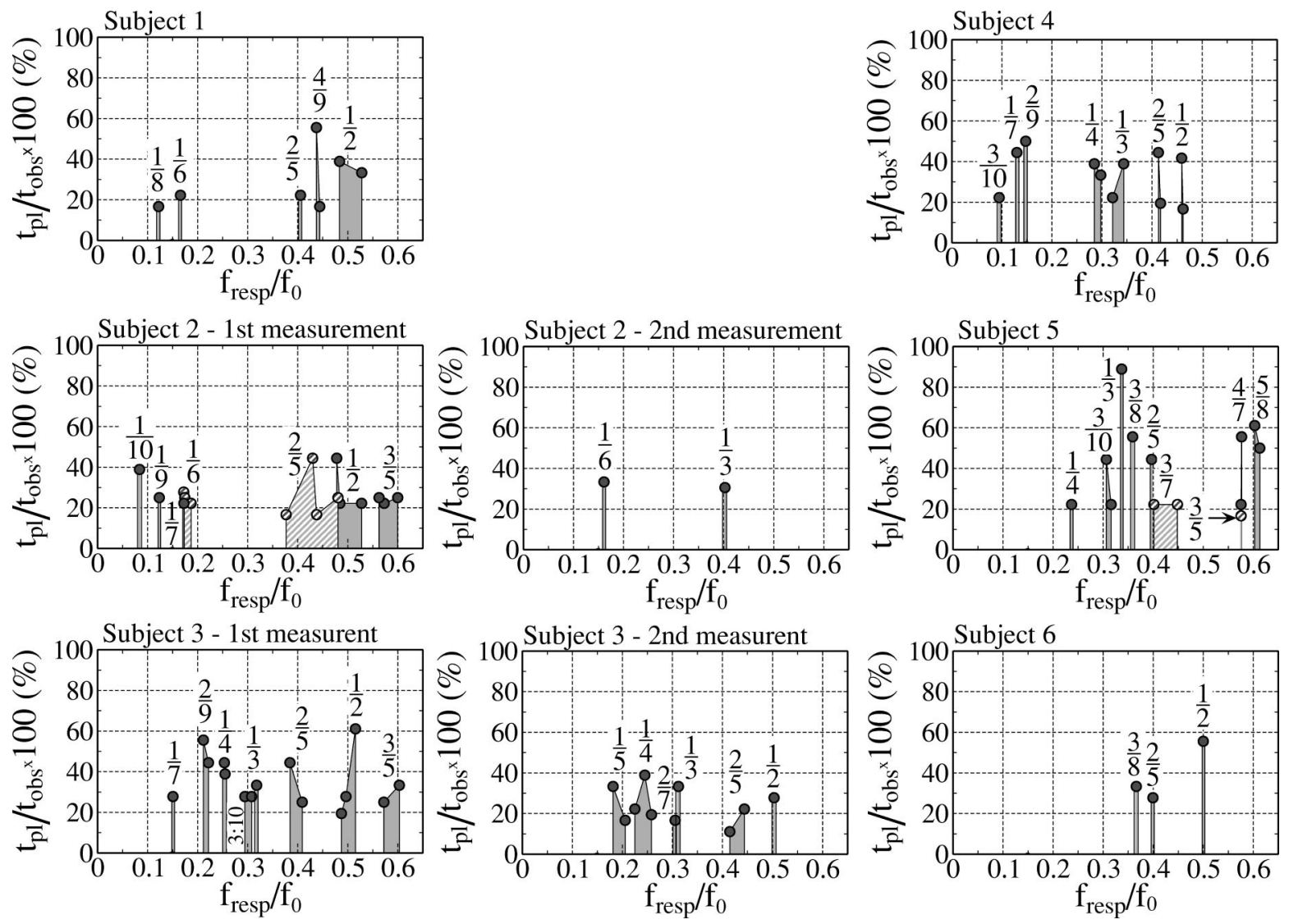

FIG. 9. Relative durations of the plateaus of phase difference $\Delta \varphi(t)$ for the given frequency ratio $f_{\text {resp }} / f_{0}$ (see text). The order of synchronization $n: m$ is marked above or near the corresponding region.

ages of the subjects. Nevertheless, several features of the results already seem clear. In particular,

(1) All six of the subjects studied demonstrated an ability to synchronize their cardiorhythm through paced respiration.

(2) Each subject studied revealed between three and nine distinct synchronization regions.

(3) For about half of the detected $n: m$ synchronization regimes the existence of an individual synchronization region was indicated by two or more data points.

(4) No obvious rule governing the width of the tongue could be inferred. Contrary to theoretical predictions, at least
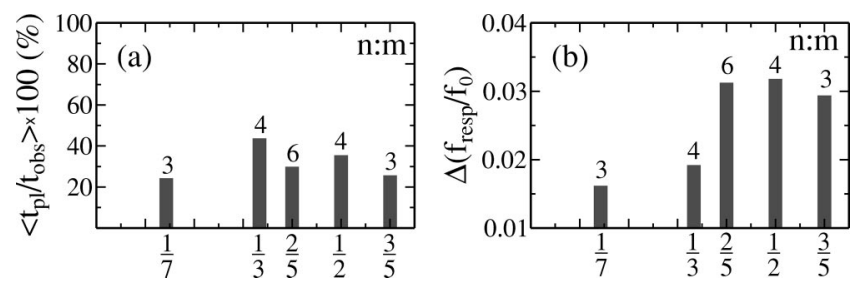

FIG. 10. (a) Effectiveness of the more common orders of synchronization $n: m$, estimated as the average duration of the plateaus of phase difference $\Delta \varphi(t)$. Averaging is made over all subjects and all datapoints within each synchronization region for the corresponding $n: m$. (b) Average width of the synchronization region for the same $n: m$ as in (a). Averaging is taken over all subjects. The number of subjects involved in each average is given above the corresponding bar. Details are given in the text. for weak resonances, the widths of the synchronization tongues detected are not inversely proportional to the denominator $m$ of synchronization order $n: m$.

(5) For a suitable choice of breathing frequency, 2:5 cardiorespiratory synchronization seems likely to be achieved in all healthy subjects. The 1:3 synchronization, which was achieved in $2 / 3$ of all cases, tends to have the epochs of longest duration.

We believe that besides their practical importance, the results obtained may be also valuable for modeling purposes. In particular, if one creates a model of the cardiorespiratory interaction, its properties can be compared with the experimental observations of the structure of the synchronization tongues. For example, it will be interesting to compare our results with a recent model [36] in order to test its applicability to the human CVS.

\section{ACKNOWLEDGMENTS}

We are grateful to Aneta Stefanovska and Janko Petrovic for help in designing, building, and installing the experimental setup, to Igor Kaufman and Andriy Bandrivskiy for writing the program for data acquisition, and to all the volunteers who participated in the measurements. The work was supported by the Engineering and Physical Sciences Research Council (UK), the Leverhulme Trust (UK), and INTAS (under Grant No. 01-2061). 
[1] A. Angelone and N. A. Coulter, J. Appl. Physiol. 19, 479 (1964).

[2] J. Brener and D. Hothersall, Psychophysiology 4, 1 (1967).

[3] M. W. Headrick and F. K. Graham, J. Exp. Psychol. 79, 486 (1969).

[4] H. Bettermann, P. Engelke, P. Van Leeuwen, and C. Heckmann, Biomed. Tech. 41, 319 (1996).

[5] C. Hayashi, Nonlinear Oscillations in Physical Systems (McGraw-Hill, New York, 1964).

[6] I. I. Blekhman, Synchronization of Dynamical Systems (Nauka, Moscow, 1971), in Russian; Synchronization in Science and Technology (ASME Press, New York, 1988).

[7] A. Pikovsky, M. Rosenblum, and J. Kurths, Synchronization: A Universal Concept in Nonlinear Science (Cambridge University Press, Cambridge, 2001).

[8] V. S. Anishchenko, V. V. Astakhov, A. B. Neiman, T. E. Vadivasova, and L. Schimansky-Geier, Nonlinear Dynamics of Chaotic and Stochastic Systems (Springer, Berlin, 2002).

[9] E. Mosekilde, Yu. Maistrenko, and D. Postnov, Chaotic Synchronization, Applications to Living Systems, Series A, Vol. 42 (World Scientific, Singapore, 2002).

[10] V. A. Kuz'menko, A. M. Badanova, and I. M. Syrkina, Hum. Physiol. 6, 936 (1980).

[11] C. Schäfer, M. G. Rosenblum, J. Kurths, and H.-H. Abel, Nature (London) 392, 239 (1998).

[12] M. Bračič-Lotrič and A. Stefanovska, Physica A 283, 451 (2000)

[13] E. Toledo, S. Akselrod, I. Pinhas, and D. Aravot, Med. Eng. Phys. 24, 45 (2002).

[14] V. M. Pokrovski, V. G. Abushkevich, A. I. Deshkovski, V. V. Skibitski, and I. A. Diak, Kardiologiia, 31, 74 (1991).

[15] A. Stefanovska, H. Haken, P. V. E. McClintock, M. Hožič, F. Bajrovič, and S. Ribarič, Phys. Rev. Lett. 85, 4831 (2000).

[16] S. Akselrod, D. Gordon, J. B. Madwed, N. C. Snidman, D. C. Shannon, and R. J. Cohen, Am. J. Physiol. 249, H867 (1985).

[17] M. V. Pitzalis, F. Mastropasqua, F. Massari, A. Passantino, R. Colombo, A. Mannarini, C. Forleo, and P. Rizzon, Cardiovasc. Res. 38, 332 (1998).

[18] M. Clark and R. Hirschman, Biofeedback Self Regul. 15, 273 (1990)

[19] M. Clark and R. Hirschman, J. Dent. Res. 59, 1533 (1980).

[20] R. R. Freedman and S. Woodward, Am. J. Obstet. Gynecol. 167, 436 (1992).

[21] P. Z. Zhang, W. N. Tapp, S. S. Reisman, and B. H. Natelson,
I\&EC Process Des. Dev. 44, 321 (1997).

[22] M. Schiek, F. R. Drepper, R. Engbert, H.-H. Abel, and K. Suder, Transition Between Two Different Cardiorespiratory Synchronization Regimes During Paced Respiration (Jahreskongress der DPG, Rostock, 1997), Vol. 76.

[23] A. V. Pomortsev, A. A. Zubakhin, V. G. Abdushkevitch, and L. F. Sedunova, in Proceedings of XVII Congress of Physiologists of Russia, 1998, edited by G. A. Kuraev (Rostov State University, Rostov, 1998), p. 316.

[24] M. Schiek, F. Drepper, H. Halling, K. Markus, M. Zarse, D. Nguyen, C. Stellbrink, P. Hanrath, T. Peschgens, and H. Hörnchen (unpublished).

[25] V. I Arnold, Geometrical Methods in the Theory of Ordinary Differential Equations (Springer, New York, 1983).

[26] The statement that the larger $m$ is, the narrower the tongue is [25], has been rigorously proved for $m>4$, that is, for weak resonances. There are no proven regularities as to the tongue width for strong resonances, that is, with $m \leqslant 4$. However, experiment and numerical simulations demonstrate that, as a rule, the same law also applies for strong resonances as well.

[27] V. S. Anishchenko, Dynamical Chaos-Models and Experiments, Appearance Routes and Structure of Chaos in Simple Dynamical Systems Series A, Vol. 8 (World Scientific, Singapore, 1995).

[28] L. Schimansky-Geier, V. S. Anishchenko, and A. Neiman, in Handbook of Biological Physics, edited by F. Moss and S. Gielen (Elsevier Science B.V., Amsterdam, 2001), Vol. 4.

[29] R. L. Stratonovich, Topics in the Theory of Random Noise (Gordon and Breach, New York, 1967), Vol. 2.

[30] T. E. Vadivasova, A. G. Balanov, O. V. Sosnovtseva, D. E. Postnov, and E. Mosekilde, Phys. Lett. A 253, 66 (1999).

[31] A. N. Malakhov, Fluctuations in Self-Oscillatory Systems (Nauka, Moscow, 1968).

[32] J. P. Saul, B. D. Berger, M. H. Chen, and R. J. Cohen, Am. J. Physiol. 256, H142 (1989).

[33] V. S. Anishchenko, A. G. Balanov, N. B. Janson, N. B. Igosheva, and G. V. Bordjugov, Int. J. Bifurcation Chaos Appl. Sci. Eng. 10, 2339 (2000).

[34] A. S. Pikovsky, M. G. Rosenblum, G. V. Osipov, and J. Kurths, Physica D 104, 219 (1997).

[35] F. Takens in Dynamical Systems and Turbulence, edited by D. A. Rang and L. S. Young, Lecture Notes in Mathematics Vol. 898 (Springer, Berlin, 1981), p. 366.

[36] K. Kotani, K. Takamasu, Y. Ashkenazy, H. E. Stanley, and Y. Yamamoto, Phys. Rev. E 65, 051923 (2002). 